\title{
Manufactured Solutions for Verification of a Coupled Flow and Material Response Code
}

\author{
Jonathan E. Wiebenga* and Iain D. Boyd ${ }^{\dagger}$ \\ University of Michigan, Ann Arbor, Michigan, 48109
}

The Method of Manufactured Solutions is used to perform order of accuracy code verification tests on some features of a hypersonic computational fluid dynamics (CFD) code and a material response code. The CFD verification tests include results for a two species gas mixture in thermal nonequilibrium, and the material response results are for an anisotropic material. After verifying the two codes separately, a verification test is performed with the codes loosely coupled so that the material response code provides updated boundary conditions to the CFD code. In the cases tested, the observed order of accuracy of the CFD code is less than the theoretical order. The tests of the material response code, however, show the expected order of accuracy.

Nomenclature

$C_{h} \quad$ Aerodynamic heat transfer coefficient

E Energy per unit volume

$J \quad$ Species diffusion flux

$N_{s} \quad$ Number of flow field species

$R \quad$ Radius

$T \quad$ Temperature

$\delta_{i j} \quad$ Kronecker delta

$\dot{Q} \quad$ Material response source term

$\dot{\omega}_{s} \quad$ Chemical source term

$\dot{\omega}_{v} \quad$ Vibrational energy source term

$\dot{m} \quad$ Mass flux

$\dot{s} \quad$ Surface recession rate

$\epsilon \quad$ Emissivity

$\hat{\mathrm{E}} \quad$ Error

$\overline{\mathbf{k}}, k_{i j} \quad$ Thermal conductivity tensor

n Normal vector

q, $q$ Heat flux vector, heat flux

v Velocity

$\rho \quad$ Density

$\sigma \quad$ Stefan-Boltzmann constant

$\tau_{i j} \quad$ Viscous stress tensor

$\begin{array}{ll}c_{p} & \text { Specific heat } \\ e & \text { Specific energy } \\ h & \text { Specific enthalpy } \\ k & \text { Trajectory point index } \\ n & \text { Number of flow field iterations before calling } \\ & \text { material response } \\ p & \text { Pressure, order of accuracy } \\ q C F D & \text { Heat flux from CFD } \\ r & \text { Grid refinement ratio } \\ u, v, w & \text { Velocity components } \\ S u b s c r i p t s \\ \infty & \text { Free stream quantity } \\ a b l & \text { Ablation quantity } \\ a h & \text { Aerodynamic heating } \\ c & \text { Char quantity } \\ c s & \text { Control surface } \\ r & \text { Recovery quantity } \\ r o t & \text { Rotational } \\ s & \text { Species } \\ t o t & \text { Total quantity } \\ t r & \text { Translational-rotational } \\ v e & \text { Vibrational-electronic } \\ w & \text { Wall quantity } \\ & \end{array}$

\section{Introduction}

YOMPUTATIONAL tools have become increasingly more important and more prevalent in the design and $\checkmark$ analysis of aerospace vehicles. In addition, as computers have become more powerful, the scale and complexity of problems that can be investigated using computational tools has grown rapidly. One area

\footnotetext{
* Graduate Student, Department of Aerospace Engineering, AIAA Student Member.

${ }^{\dagger}$ Professor, Department of Aerospace Engineering, Fellow AIAA
} 
where this is true is the computation of hypersonic flow fields, and the coupled analysis of the flow and the thermal response of a vehicle during high speed flight. This is a difficult problem involving many complex physical phenomena such as: chemical and thermal nonequilibrium, reacting flows, strong shocks, surface chemistry, ablation, anisotropic materials, nonlinear coupling, and others. When developing computational codes, it is important to ensure that the code is working as expected and that there are no "bugs" or other implementation errors that may affect the results. This becomes especially important, and especially difficult, for codes dealing with complex physical phenomena.

The process of ensuring that the equations being solved by a code are being solved correctly and that there are no implementation errors affecting the results is known as verification. Verification and validation are two terms that are often used interchangeably when talking about code development, however, in reality, they refer to two separate but equally important ideas. Validation is the process of ensuring that the models used in a code accurately reflect the physical process being modeled. This can be done by comparing computational results with experimental data. Verification, on the other hand, is concerned with whether or not the models used in a code have been correctly implemented, and whether the theoretical order of accuracy of the code can be obtained. Therefore, verification is a purely mathematical exercise and does not require experimental data or even physically realizable solutions to the governing equations.

There are several ways in which verification can be accomplished including: comparison with exact analytical solutions, comparison with highly refined solutions from numerical benchmarks, code-to-code comparisons, and the Method of Manufactured Solutions ${ }^{1}$ (MMS). Out of all of these methods, only the Method of Manufactured Solutions and comparisons with exact solutions can be considered rigorous order of accuracy verification procedures. ${ }^{2}$ Of these two methods, using manufactured solutions offers an advantage in that it does not require analytical solutions of the governing equations. This is especially useful for codes modeling complex or coupled physics when exact solutions are not available or are too limited in scope to test all of the features of a code.

This paper presents the results of several verification tests performed on two different codes using the Method of Manufactured Solutions. The first code is LeMANS: a hypersonic CFD code developed at the University of Michigan. Manufactured solutions are developed for LeMANS and used to compute the order of accuracy of the code using both far field and no-slip, isothermal wall boundary conditions. The second code that is used is a material response code also developed at the University of Michigan. Manufactured solutions are again developed for this code, and the order of accuracy is determined using both specified temperature and specified heat flux boundary conditions. Finally, LeMANS and the material response code are coupled together through boundary conditions, and manufactured solutions are used in order to compute the order of accuracy of LeMANS that results from this coupling procedure. In all cases the observed order of accuracy is compared with the theoretical order of accuracy.

\section{Code Overviews}

\section{II.A. CFD}

The CFD code used in this study is LeMANS, ${ }^{3,4}$ which is a laminar Navier-Stokes solver developed at the University of Michigan for use on hypersonic problems. It is capable of simulating flows with both thermal (translation/rotational/vibrational) and chemical non-equilibrium. The translational and rotational energy modes of all species are described by the temperatures $T$ and $T_{\text {rot }}$ respectively, and the combined vibrational and electronic energies of all species are described by a single temperature, $T_{v e}{ }^{5}$ In this work it is assumed that the translational and rotational modes are in equilibrium and can be described by a single temperature, $T_{t r}$. The governing equations solved in LeMANS are shown in Equations $1-4$.

$$
\begin{aligned}
& \frac{\partial \rho_{s}}{\partial t}+\frac{\partial}{\partial x_{i}}\left(\rho_{s} u_{i}+J_{i, s}\right)=\dot{\omega}_{s} \\
& \frac{\partial \rho u_{i}}{\partial t}+\frac{\partial}{\partial x_{i}}\left(\rho u_{i} u_{j}+p \delta_{i j}-\tau_{i j}\right)=0 \\
& \frac{\partial E_{t o t}}{\partial t}+\frac{\partial}{\partial x_{i}}\left(E_{t o t} u_{i}+p u_{i}-u_{i} \tau_{i j}+q_{t r_{i}}+q_{v e_{i}}+\sum J_{i, s} h_{s}\right)=0 \\
& \frac{\partial E_{v e}}{\partial t}+\frac{\partial}{\partial x_{i}}\left(E_{v e} u_{i}-q_{v e_{i}}+\sum J_{i, s} e_{v e, s}\right)=\dot{\omega}_{v}
\end{aligned}
$$


Viscous stresses are modeled assuming a Newtonian fluid and using Stokes' hypothesis. Transport properties are modeled using either Wilke's semi-empirical mixing rule with Blottner's curve fits for viscosity and Eucken's relation for thermal conductivities, or using Gupta's mixing rule with non-coulombic/coulombic collision cross section data to calculate viscosities and thermal conductivities. For this study the former method is used. The heat flux consists of a convective term, modeled using Fourier's law for all temperatures, and a mass diffusion term modeled using a modified form of Fick's Law. ${ }^{6}$ The surface can be treated as either non-catalytic or super-catalytic, and, in addition, ablative species can be introduced via a blowing boundary condition. ${ }^{7}$

Inviscid fluxes are computed using a modified Steger-Warming Flux Vector Splitting Scheme, ${ }^{8}$ which has low dissipation in boundary layer regions. Second order accuracy for the inviscid fluxes is obtained by using a MUSCL scheme to reconstruct the cell values. The viscous fluxes are calculated using a stencil that includes property values at both cell centers and nodes. ${ }^{9}$ This avoids loss of accuracy when using unstructured meshes and allows for second order viscous flux calculations. Time integration is performed using either a point or line implicit method.

\section{II.B. Material Thermal Response}

The material response calculations are performed using a code that is being developed at the University of Michigan, and is based on MOPAR, ${ }^{10}$ a one-dimensional material response code also developed at the University of Michigan. The material response model solves the energy equation shown in Equation 5 using Newton's Method with a preconditioned GMRES ${ }^{11}$ linear solver to solve the nonlinear energy equation. Since the model is currently designed to be used solely for surface (non-pyrolyzing) ablators, Equation 5 is the only conservation equation that is solved.

$$
\rho \frac{\partial e}{\partial t}-\rho h\left(\nabla \cdot \mathbf{v}_{c s}\right)+\nabla \cdot \mathbf{q}=\dot{Q}
$$

The Control Volume Finite-Element Method (CVFEM) ${ }^{12,13}$ with linear triangular elements is used for spatial discretization. In this formulation, all variables are assumed to vary linearly across an element, which leads to a scheme that is spatially second order. The Backward Euler method is used for implicit time integration, which leads to a first order accurate discretization in time. The heat flux is modeled using a tensor formulation of Fourier's Law to allow for simulation of anisotropic materials. The control surface velocity is determined from the change in node positions during a time step as the mesh is deformed.

Several different boundary conditions are implemented including specified temperature, specified heat flux, re-radiation, aerodynamic heating, and thermochemical ablation. If a surface is ablating, the last three boundary conditions along with Equation 5 give the energy balance at the surface shown in Equation 6 .

$$
\underbrace{-\overline{\mathbf{k}} \nabla T \cdot \hat{\mathbf{n}}}_{\text {Conduction }}-=\underbrace{C_{h}\left(h_{r}-h_{w}\right)}_{\text {Convection }}-\underbrace{\epsilon_{w} \sigma\left(T_{w}^{4}-T_{\infty}^{4}\right)}_{\text {Re-radiation }}-\underbrace{\dot{m}_{a b l}\left(h_{w}-h_{c}\right)}_{\text {Ablation }}
$$

The wall enthalpy and ablative mass flux can be obtained either from interpolating a thermochemical $\left(B^{\prime}\right)$ table which lists these quantities as functions of pressure and temperature, or from a separate surface chemistry code which is coupled to the material response code. ${ }^{14}$ The surface recession rate can then be found from $\dot{m}_{a b l}$ using Equation 7 .

$$
\dot{s}=\frac{\dot{m}_{a b l}}{\rho_{c}}
$$

To move the solution forward a time step when there is ablation, the method of Hogan, Blackwell, and Cochran ${ }^{15}$ is used to iteratively calculate $\dot{s}$, compute new temperatures, and move the mesh if necessary. This method iterates the thermal and mesh motion solutions at each time step until the change in surface temperature during that time step falls below some desired level. If there is no ablation, then this iterative method is not needed and the ablation term in Equation 6 is not computed.

\section{II.C. Coupling}

The material response model can be implemented as a boundary subroutine of LeMANS in order to link the flow and solid calculations. This method of coupling CFD and material response has been demonstrated for LeMANS using both one-dimensional ${ }^{7}$ and multidimensional ${ }^{16}$ material response codes. In this method, 
a vehicle's trajectory is discretized into several points. A steady state flow solution is then found at each trajectory point using the solution from the previous trajectory point as the initial condition. As the CFD solution of the flow field is marched to steady state, the material response subroutine is called after every $n$ flow field iterations, where $n$ is a fixed number selected before running the simulation.

Depending on the surface chemistry model being used and whether or not ablation is being considered, the boundary values passed from LeMANS may be different. In cases where ablation is being modeled using a $B^{\prime}$ table, then the aerodynamic heating boundary condition shown in Equation 8 is used to compute the convective heat flux, and the heat flux from LeMANS is used to compute $C_{h}$ from Equation $9 .{ }^{17}$

$$
\begin{gathered}
q_{a h}=\rho C_{h}\left(h_{w}-h_{r}\right) \\
C_{h}=\frac{q_{C F D}}{h_{r}-h_{w}}
\end{gathered}
$$

In cases where there is no ablation or ablation is modeled using a surface chemistry subroutine in LeMANS, then $q_{a h}=q_{C F D}$ and Equation 9 is not needed. Additionally, the wall enthalpy and ablative mass flux are passed from LeMANS rather than calculated via a $B^{\prime}$ table.

Once the boundary condition is specified, the material response is then modeled in a time-accurate fashion from trajectory point $k$ to $k+1$ with the applied heat flux and other boundary variables being linearly interpolated from the initial condition to the current value calculated by LeMANS. Once the material response has been modeled up to trajectory point $k+1$, the updated wall temperature, wall position, and mass flux (if using a $B^{\prime}$ table) are then passed back to LeMANS, and the flow field is iterated for $n$ more iterations before the material response is called again. A diagram of the coupling procedure is shown in Figure 1.

In order to provide flexibility when generating meshes, the nodes of the fluid and solid domains at their interface are not required to be coincident. So while the surface shape will be the same in both meshes, the number elements can be different. This allows for independent refinements of the flow and solid meshes tailored to the problem that is being solved. One downside of this approach, however, is that it is necessary to interpolate values between the two meshes. To accomplish this, a version of Shepard's method ${ }^{18,19}$ is used. This is an inverse-distance weighting method that uses data within some radius, $R$, of the location where an interpolated value is desired. The initial search radius is increased until a user-defined minimum number of interpolating points is found. This method is very general and can easily be extended to three-dimensional geometries.

\section{Manufactured Solutions}

One of the most thorough approaches to verifying a numerical code is to check the order of accuracy of the code. If the observed order of accuracy does not match the expected order of accuracy, then there is a strong possibility that an error is present. The ob-

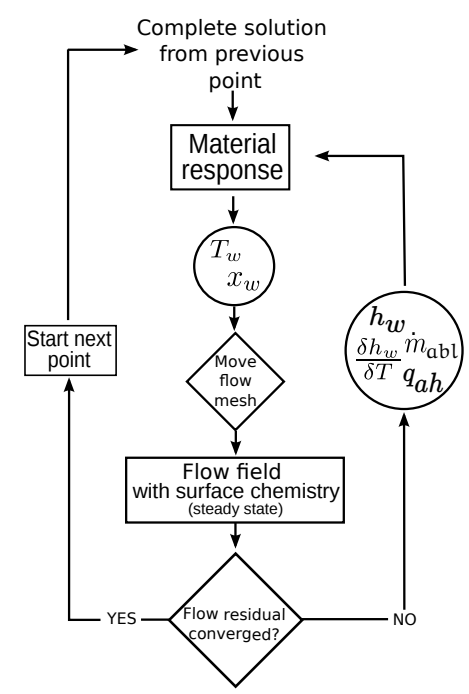

Figure 1: Diagram of the fluid/material response coupling using a surface chemistry subroutine in LeMANS.

served order of accuracy can be determined from Equation 10 where $\hat{\mathrm{E}}\left(\right.$ grid $\left._{1}\right)$ and $\hat{\mathrm{E}}\left(\right.$ grid $\left._{2}\right)$ are measures of error on two separate grids, and $r$ is the refinement ratio between the grids. If, after a series of systematic grid refinements, the observed order of accuracy is less that expected, then it is likely that an error exists in the code.

$$
p=\frac{\log \left(\frac{\hat{\mathrm{E}}\left(\text { grid }_{1}\right)}{\hat{\mathrm{E}}\left(\text { grid }_{2}\right)}\right)}{\log (r)}
$$

Order of accuracy verification can be performed using analytical solutions to the governing equations that are solved in the code, but this approach has its limits. In order to fully verify a code, all of the different features of the code must be tested. Often there are no analytical solutions that exercise all the features of a code and all of the terms in the governing equations. This is where the Method of Manufactured Solutions can be useful. 
The Method of Manufactured Solutions is a powerful tool for performing order of accuracy verification of a numerical code that does not require analytical solutions to the governing equations being solved. The basic idea is to choose, or "manufacture", analytical solutions for each dependent variable, and then compute analytical source terms to balance the governing equations. The source term is necessary since the manufactured solutions do not in general satisfy the governing equations on their own. Thankfully, while the source term may be a complicated function, it is found in a straightforward fashion by applying the governing equations to the manufactured solutions. This method has been used in several different verification exercises including verification of a material response $\operatorname{code}^{20}$ and Euler and Navier-Stokes codes. ${ }^{21-23}$

In this work, the method of manufactured solutions is used to verify LeMANS and a material response code independently, and then look at the order of accuracy of the two codes when they are coupled. Looking first at LeMANS, it is necessary based on equations 1 - 4 to construct solutions for $N_{s}+5$ variables (where $N_{s}$ is the number of species) that are independent of one another. For this work, a two species flow model consisting of atomic and molecular nitrogen is used. Therefore, the set of variables for which solutions are manufactured is $\rho_{N_{2}}, \rho_{N}, u, v, w, T_{t r}, T_{v e}$. Note that this is not the only valid set of variables that could be used, but represents one possible convenient set. In addition to the governing equations, the necessary auxiliary relations for thermodynamic and transport properties, and the necessary vibrational energy constants for $\mathrm{N}$ and $\mathrm{N}_{2}$ can be found in Scalabrin. ${ }^{3}$ While LeMANS is able to simulate chemically reacting flows, that capability is not tested in this work and it is assumed that there is no $\mathrm{N}_{2} / \mathrm{N}$ chemistry.

For the material response code, the governing equation is Equation 5, so the only variable that needs to be analytically defined is temperature. In addition, analytical forms of the specific heat and thermal conductivity are constructed.

Several guidelines for defining solutions to these variables are discussed in Knupp and Salari. ${ }^{1}$ A Solution should be smooth enough to allow the theoretical order of accuracy to be obtained, contain enough nontrivial derivatives to truly test the code, and be general enough that it exercises all terms in the governing equations. In addition, since LeMANS contains a limiter, it is desirable to initially develop solutions that are smooth enough that the limiter does not impact the observed order of accuracy. Finally, the solution should be chosen so that code robustness is not an issue.

Based on these guidelines, the manufactured solutions for LeMANS are shown in Equation 11 and the manufactured solution for the material response code is shown in Equation 12. The constructed values for thermal conductivity and specific heat for the material response code are shown in Equation 13. The constants $\left(B, \omega, \epsilon, k_{0}, k_{1}, k_{2}, c_{p_{0}}, c_{p_{1}}\right)$ in each of these equations can be adjusted to modify both the difficulty of the problem and also the portions of the code that are being tested. For example, by changing the values of different constants in Equation 11 it is possible to simulate 1D, 2D, 3D, time-varying, and steady state problems. The work presented in this paper focuses on 2D, steady-state problems in LeMANS, so $\omega_{\theta}=B_{\theta y}=0.0$ for all LeMANS equations. Verification for the material response code focuses on 2D, time-varying problems, so $B_{y}=0.0$ in Equation 12 .

$$
\begin{gathered}
\rho_{s}=\rho_{s_{0}} \cos \left(B_{s x} x^{2}+B_{s y} y^{2}+B_{s z} z^{2}+\omega_{\rho_{s}} t\right)+\epsilon_{\rho_{s}} \\
\left.\begin{array}{c}
u=u_{0} \sin \left(B_{u x} x^{2}+B_{u y} y^{2}+B_{u z} z^{2}+\omega_{u} t\right)+\epsilon_{u} \\
v=v_{0} \sin \left(B_{v x} x^{2}+B_{v y} y^{2}+B_{v z} z^{2}+\omega_{v} t\right)+\epsilon_{v} \\
w=w_{0} \sin \left(B_{w x} x^{2}+B_{w y} y^{2}+B_{w z} z^{2}+\omega_{w} t\right)+\epsilon_{w} \\
T_{t r}=T_{T_{t r_{0}}} \cos \left(B_{T_{t r} x} x^{2}+B_{T_{t r} y} y^{2}+B_{T_{t r} z} z^{2}+\omega_{T_{t r}} t\right)+\epsilon_{T_{t r}} \\
T_{v}=T_{v_{0}} \cos \left(B_{T_{v} x} x^{2}+B_{T_{v} y} y^{2}+B_{T_{v} z} z^{2}+\omega_{T_{v}} t\right)+\epsilon_{T_{v}}
\end{array}\right\} \\
\left.\begin{array}{c}
T=T_{0} \cos \left(B_{x} x^{2}+B_{y} y^{2}+B_{z} z^{2}+\omega_{T} t\right)+\epsilon_{T} \\
k_{i j}=k_{0_{i j}}+k_{1_{i j}} T+k_{2_{i j}} T^{2} \\
c_{p}=c_{p_{0}}+c_{p_{1}} T
\end{array}\right\}
\end{gathered}
$$

One especially convenient aspect of MMS is that the same solutions can be used with a variety of different boundary conditions. In this work, far field and no-slip, isothermal wall boundary conditions are tested in LeMANS, and specified temperature and specified heat flux boundary conditions are tested in the material response code. For the far field, specified temperature, and specified heat flux boundary conditions, the manufactured solutions shown in Equations 11 and 12 can be used without modification. The wall 
boundary condition, however, requires a surface to be defined in the manufactured solution such that, for a no-slip, isothermal wall, the velocity is zero and the temperature is constant on the surface. The modified manufactured solutions used to enforce this boundary condition are shown in Equation 14. In this work, $f(x, y, z)=y$ and $C_{s}=0.0$ which enforces zero velocity and constant temperature at a boundary located at $y=0.0$.

$$
\begin{aligned}
u & =\left(f(x, y, z)-C_{s}\right)\left\{u_{0} \sin \left(B_{u x} x^{2}+B_{u y} y^{2}+B_{u z} z^{2}+\omega_{u} t\right)+\epsilon_{u}\right\} \\
v & =\left(f(x, y, z)-C_{s}\right)\left\{v_{0} \sin \left(B_{v x} x^{2}+B_{v y} y^{2}+B_{v z} z^{2}+\omega_{v} t\right)+\epsilon_{v}\right\} \\
w & =\left(f(x, y, z)-C_{s}\right)\left\{w_{0} \sin \left(B_{w x} x^{2}+B_{w y} y^{2}+B_{w z} z^{2}+\omega_{w} t\right)+\epsilon_{w}\right\} \\
T_{t r} & =\left(f(x, y, z)-C_{s}\right) T_{T_{t r_{0}}} \cos \left(B_{T_{t r} x} x^{2}+B_{T_{t r} y} y^{2}+B_{T_{t r} z} z^{2}+\omega_{T_{t r}} t\right)+\epsilon_{T_{t r}} \\
T_{v} & =\left(f(x, y, z)-C_{s}\right) T_{v_{0}} \cos \left(B_{T_{v} x} x^{2}+B_{T_{v} y} y^{2}+B_{T_{v} z} z^{2}+\omega_{T_{v}} t\right)+\epsilon_{T_{v}}
\end{aligned}
$$

Once the manufactured solutions have been constructed, it is necessary to generate analytical source terms for each of the governing equations. As mentioned previously, the source terms serve to balance the equations since, in general, the manufactured solutions are not physically valid solutions of the governing equations. This is performed by substituting the manufactured solutions into the governing equations and computing the exact result. This generally leads to very complicated source terms, so, in order to save time and reduce the possibility for algebraic errors, Mathematica ${ }^{24}$ is used to compute the source terms. This is convenient as well because Mathematica can output the results directly as code for use in a C or FORTRAN program.

\section{Results}

\section{IV.A. LeMANS}

The Method of Manufactured Solutions is applied to LeMANS using two different sets of boundary conditions, the first of which is the far field boundary condition. For this boundary condition, values of the conserved quantities are computed from the manufactured solutions and enforced at the boundary cells. Since steady state solutions are being sought, these values are constant throughout the simulation. Three different cases are tested using the far field boundary condition. Figure 2 shows the Mach number contours for the first two cases. These cases are identical to one another except for the magnitude of the u-velocity component and are meant to be simple initial test cases. Case 1 is subsonic everywhere and case 2 is supersonic everywhere. All solutions are computed on uniform square meshes.

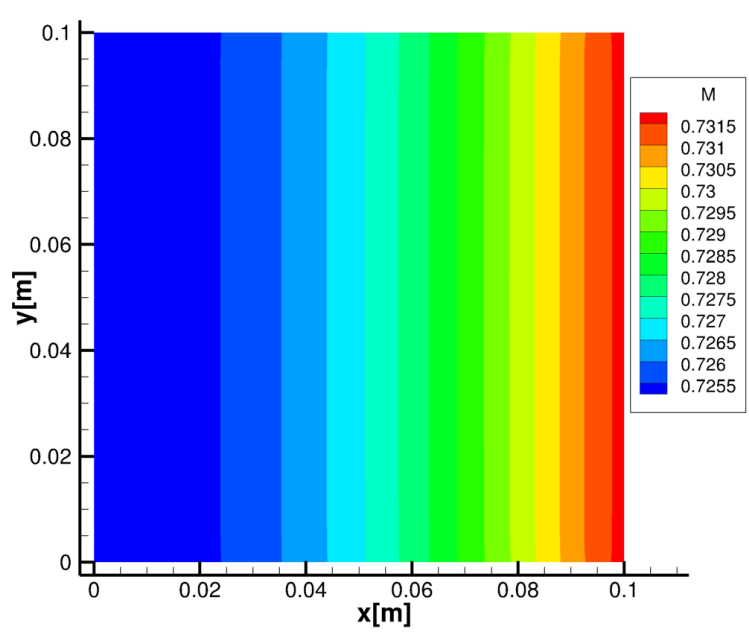

(a) Far field boundary case 1

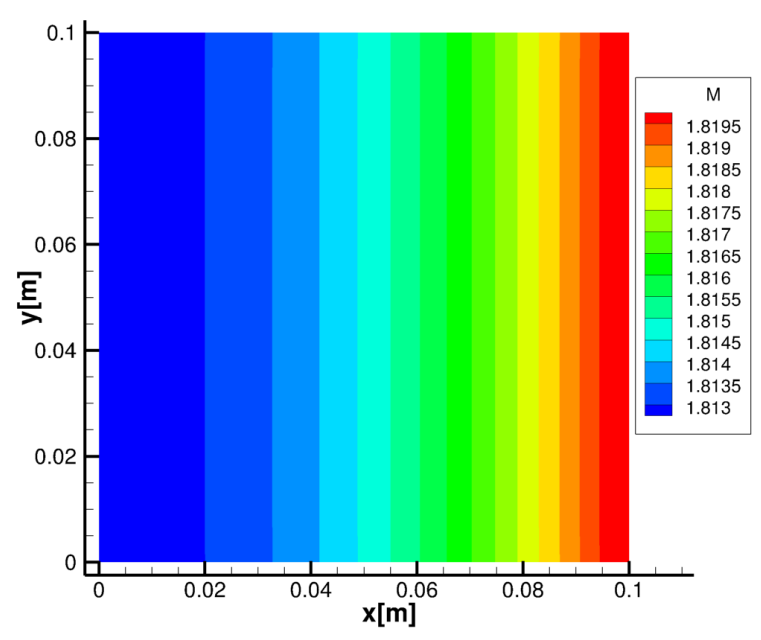

(b) Far field boundary case 2

Figure 2: Mach number contours for the first two far field boundary cases. 
The observed order of accuracy results for these two cases are shown in Figure 3. For both of these cases, the order of accuracy for most variables is less than the expected value of 2 . In addition, the order of accuracy is somewhat higher for the supersonic case than for the subsonic case.

The third case, shown in Figure 4, uses far field boundary conditions on the left and bottom boundaries, and a zeroth order extrapolation supersonic outlet boundary condition on the top and right boundaries. This is a more complicated supersonic case where all solutions vary nonlinearly in both the $\mathrm{x}$ and $\mathrm{y}$ directions. Figure 4a shows the Mach number contours for this case. Though not shown in the figure, care is taken to ensure that Mach number normal to the boundary surfaces is supersonic at all boundaries. Figure 4b shows the observed order of accuracy for this case. The order is between 1.4 and 1.5 for most variables, which is similar to the supersonic case in Figure 3.

In addition to the far field boundary condition, a no-slip, isothermal wall boundary condition is also tested. The wall boundary condition is applied at the bottom boundary of the domain, and far field boundary conditions are applied at the other three boundaries. Figure 5a shows the Mach number contours for this case. In order to test the wall boundary condition, the manufactured solutions from Equation 14 are used. The solutions are subsonic everywhere and $T_{t r}=2000 \mathrm{~K}$ and $T_{v e}=500 \mathrm{~K}$ at the wall. The observed order of accuracy for this case, shown in Figure 5b, is slightly greater than 1. Again, this is considerably less than the $2^{\text {nd }}$ order accuracy which is expected when using these boundary conditions.

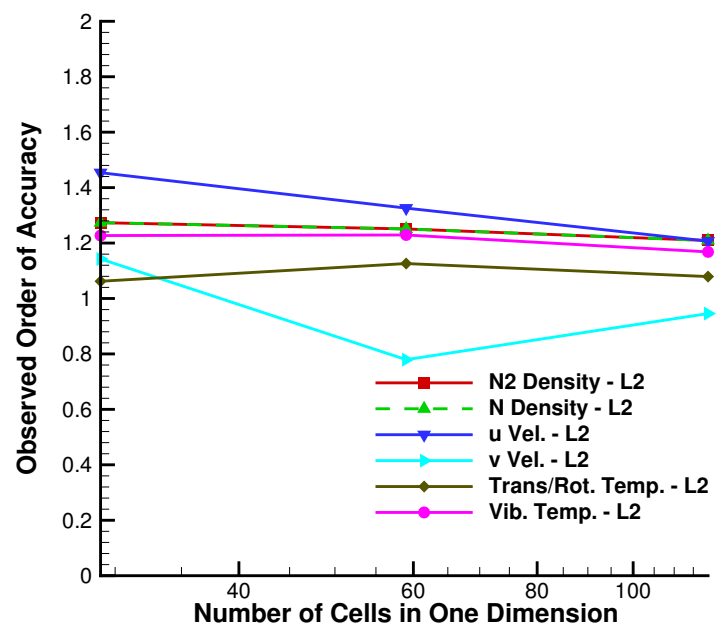

(a) Case 1 (subsonic)

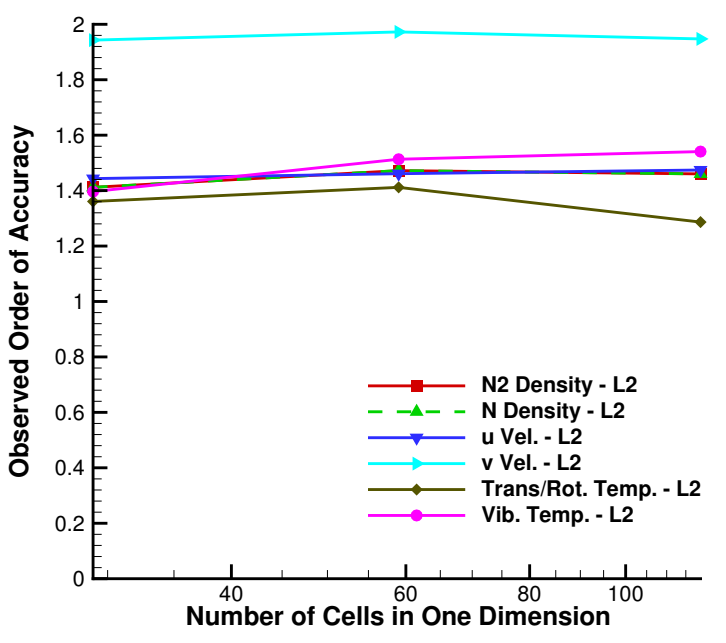

(b) Case 2 (supersonic)

Figure 3: Observed order of accuracy of LeMANS based on the $L_{2}$ norm of error for a simple case using a far field boundary condition.

All of the manufactured solution cases run in LeMANS indicate an order of accuracy between 1 and 2, usually closer to 1 . The expected order of accuracy is 2 for all cases. One possible reason for this discrepancy is the implementation of the MMS at domain boundaries. Figure 6 shows the error contours of v-velocity for the cases shown in Figures $2 \mathrm{~b}$ and $4 \mathrm{a}$. It can be seen that in both cases the largest error is present directly adjacent to one or more of the boundaries. This is true of the error contours for all other variables, and for the wall boundary condition case as well. Additionally, Figure 7 shows the order of accuracy based on the $L_{\infty}$ error norms for these two cases. The order of accuracy is considerably lower than that based on the $L_{2}$ norms, which indicates that lower order error is being generated in some area of the domain. ${ }^{23}$ This lends additional support to the idea that an error in the MMS test is leading to a reduced order of accuracy, and not an error in LeMANS. More work will be done to pinpoint the exact cause of the lower than expected order of accuracy. 


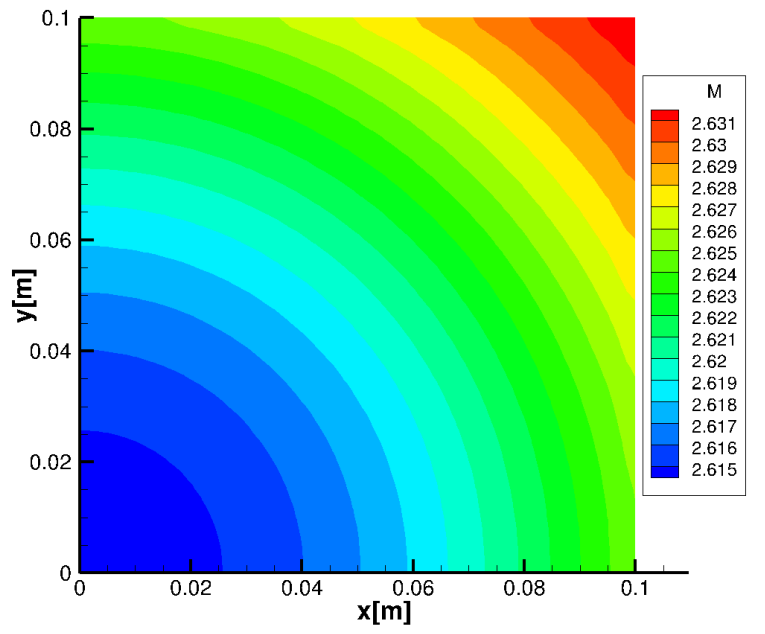

(a) Mach number contours

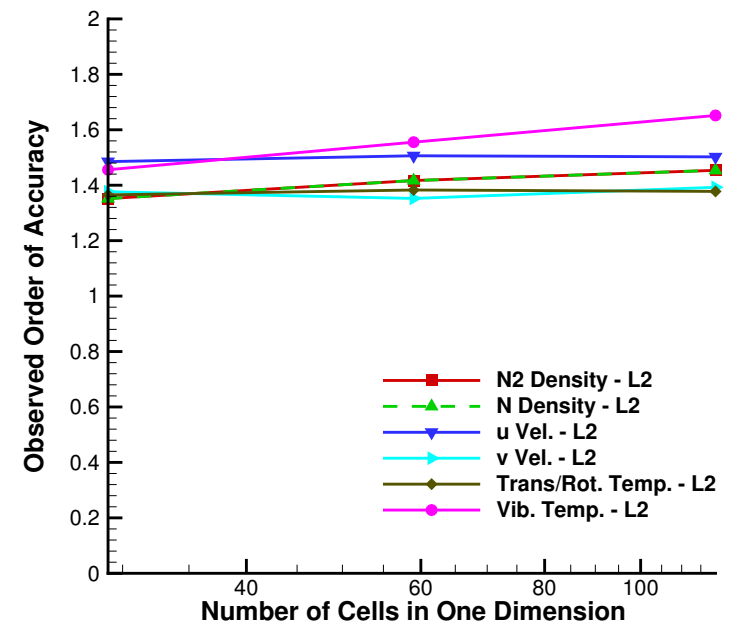

(b) Order from $L_{2}$ norm

Figure 4: Observed order of accuracy of LeMANS based on the $L_{2}$ norm of error for far field boundary conditions on left and bottom boundaries, and supersonic outflow boundary conditions on the top and right boundaries.

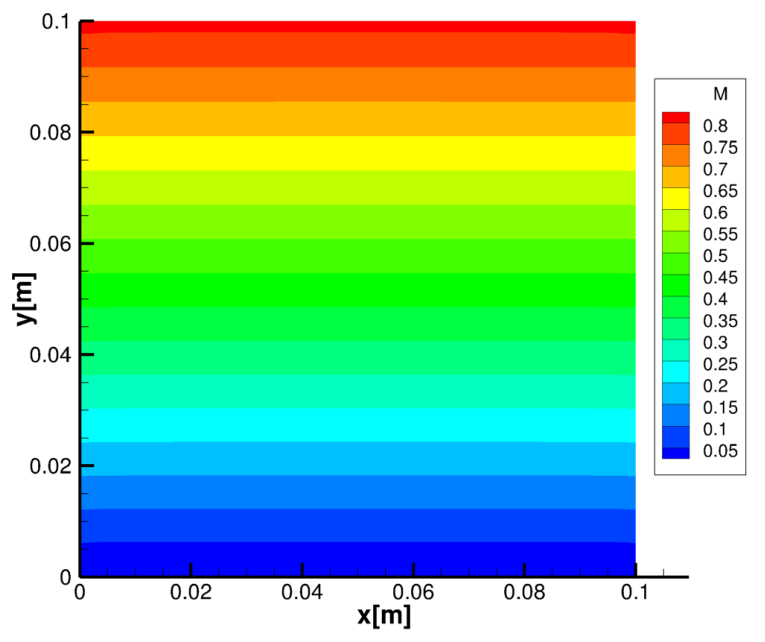

(a) Mach number contours

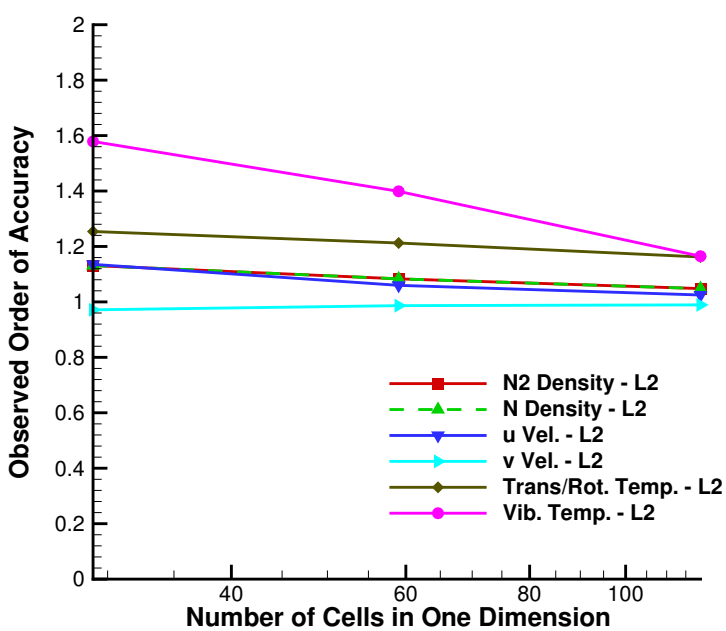

(b) Order from $L_{2}$ norm

Figure 5: Mach number contours and observed order of accuracy of LeMANS for a case with a no-slip, isothermal wall boundary condition 


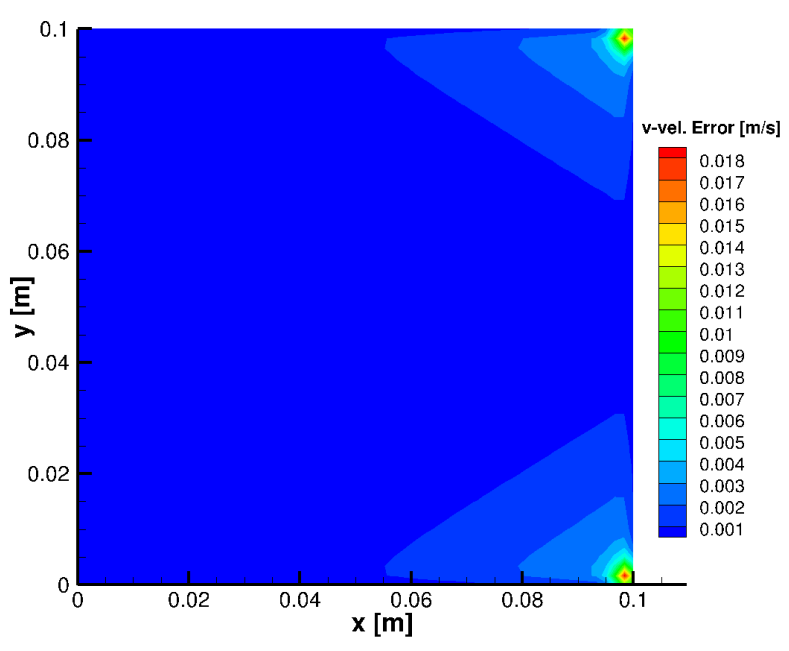

(a) Far field boundary case 2

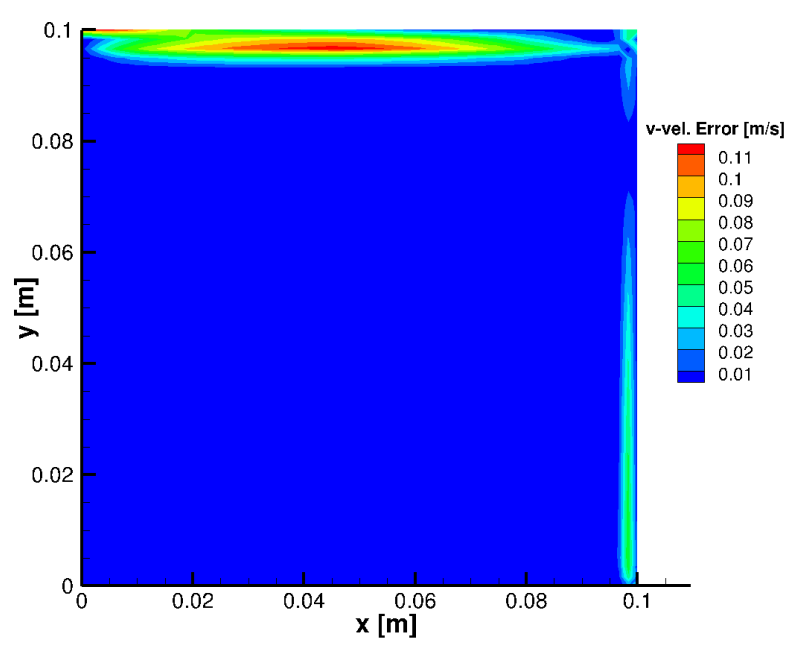

(b) Far field/supersonic outflow BC

Figure 6: Error contours of v-velocity for two different boundary condition cases.

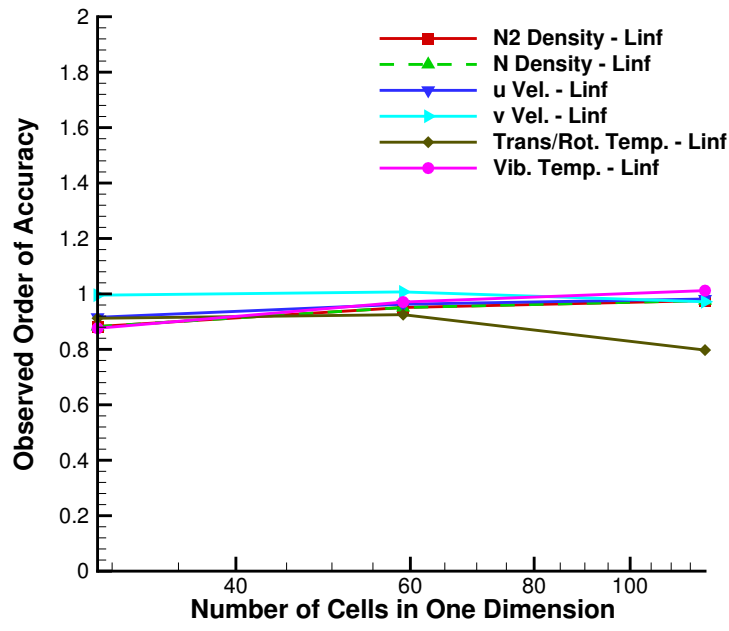

(a) Far field boundary case 2

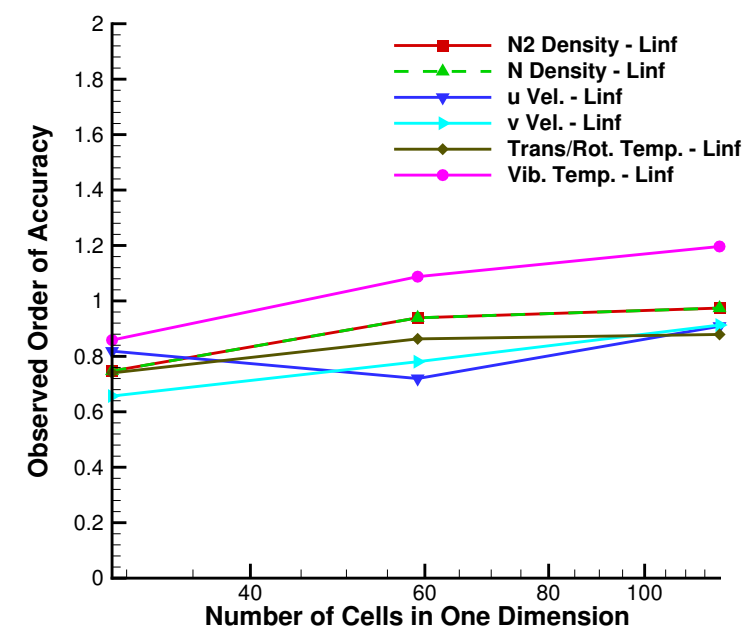

(b) Far field/supersonic outflow BC

Figure 7: Observed order of accuracy of LeMANS based on the $L_{\infty}$ norm of error for two different boundary condition cases. 


\section{IV.B. Material Response}

Time accurate results are desired for verification of the material response code, so it is important to test the order of accuracy of both the spatial discretization and the time integration. For the results presented here, spatial and temporal accuracy is simultaneously verified by adjusting both the time step and grid size at each grid refinement.

The first test case consists of specified temperature boundary conditions at all boundaries. This is a Dirichlet boundary condition and is especially useful because it allows for testing the equations on the interior cells without the added complication of boundary conditions. Similar to LeMANS, all simulations are $2 \mathrm{D}$ and run on square domains, however, in this case the mesh is unstructured. Figure 8 shows the observed order of accuracy results for this case at three different simulation times. As can be seen, the expected second order spatial accuracy and first order temporal accuracy is obtained at all three times.

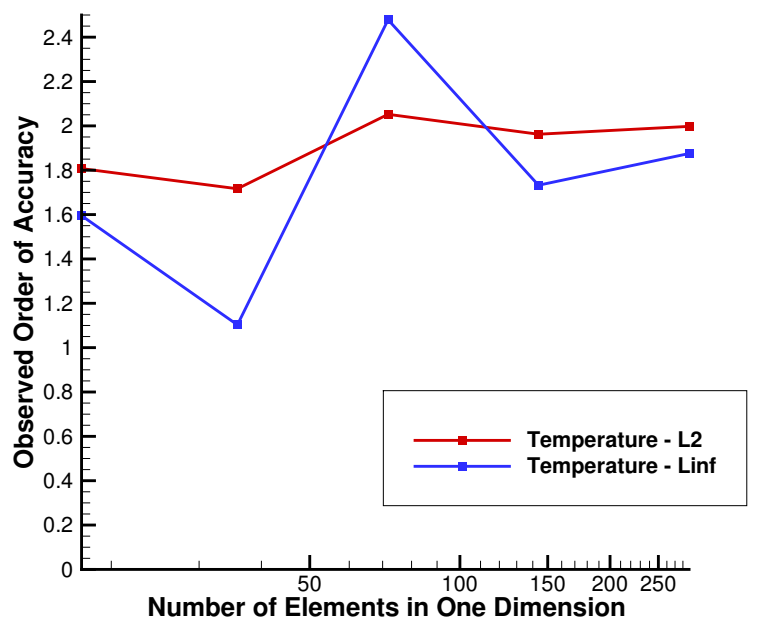

(a) 1 second

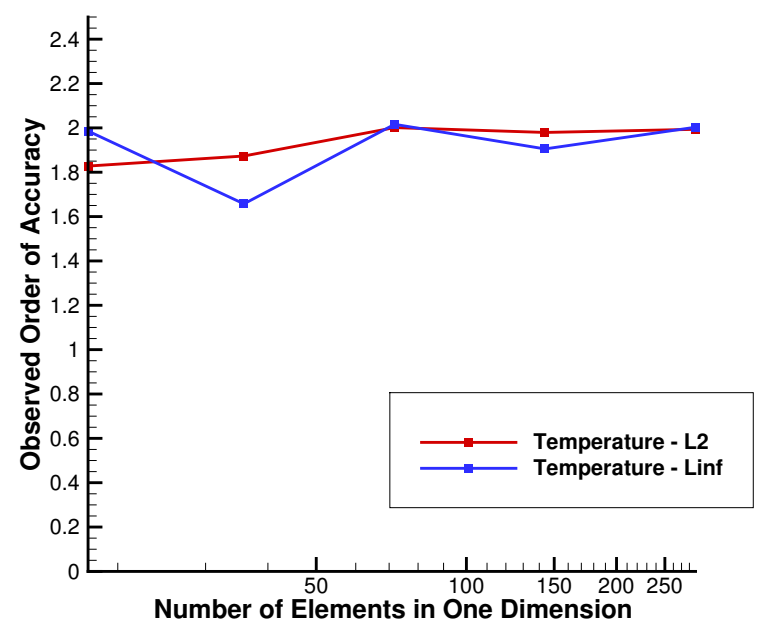

(b) 5 seconds

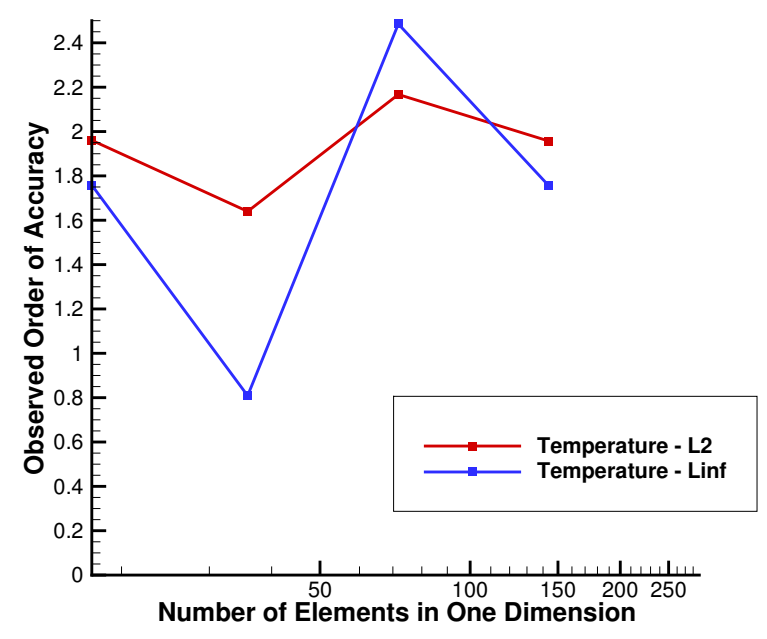

(c) Steady state (100 seconds)

Figure 8: Observed order of accuracy of material response code for three different simulation times using a specified temperature boundary condition.

The second boundary condition tested is a specified flux condition. Two different domains are used to test this boundary condition to ensure that the boundary flux calculations are correct. Figure 9 shows the 
two coarsest meshes for both domains. In both cases, a specified flux boundary condition is applied at the top boundary and a specified temperature boundary condition at the other boundaries. The skewed mesh makes for a more rigorous test case due to the more complicated boundary geometry which can be useful in identifying certain types of errors. In fact, the skewed mesh tests helped to identify an error in the computation of the surface normal vectors at specified flux boundaries. Since the time accuracy is confirmed

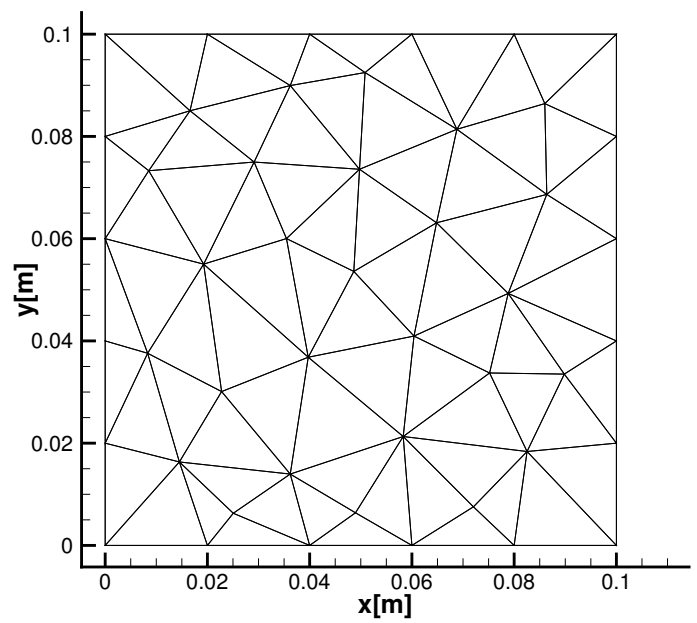

(a) Square domain

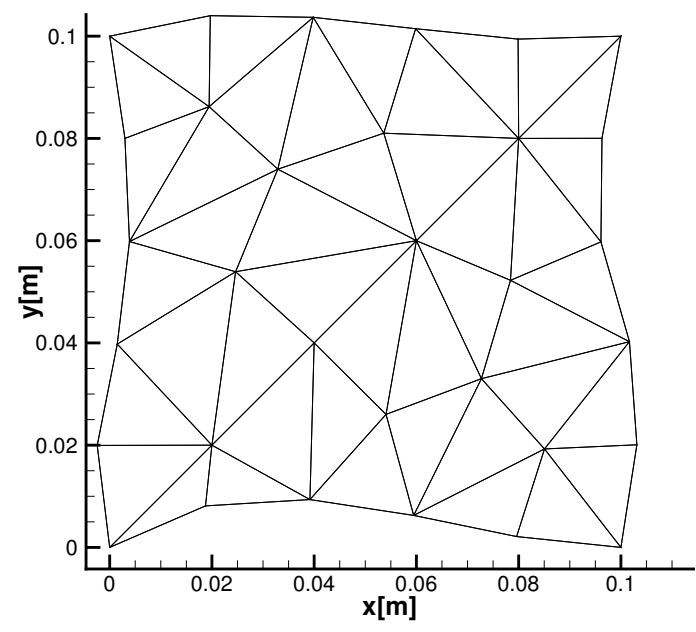

(b) Skewed domain

Figure 9: Coarse meshes used for testing the specified heat flux boundary condition for the material response code.

during the specified temperature cases, the specified flux case is tested at only one time. Order of accuracy results for this case for both domains are shown in Figure 10. As the mesh is refined, the order of accuracy from the $L_{2}$ norm again approaches second order, which is the expected result. The $L_{\infty}$ norm, however, shows a much lower order of accuracy.

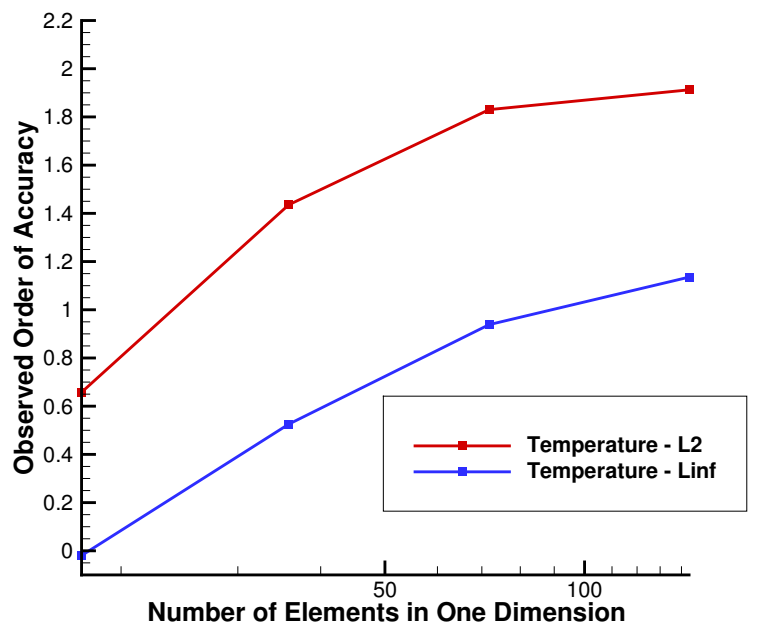

(a) Square domain

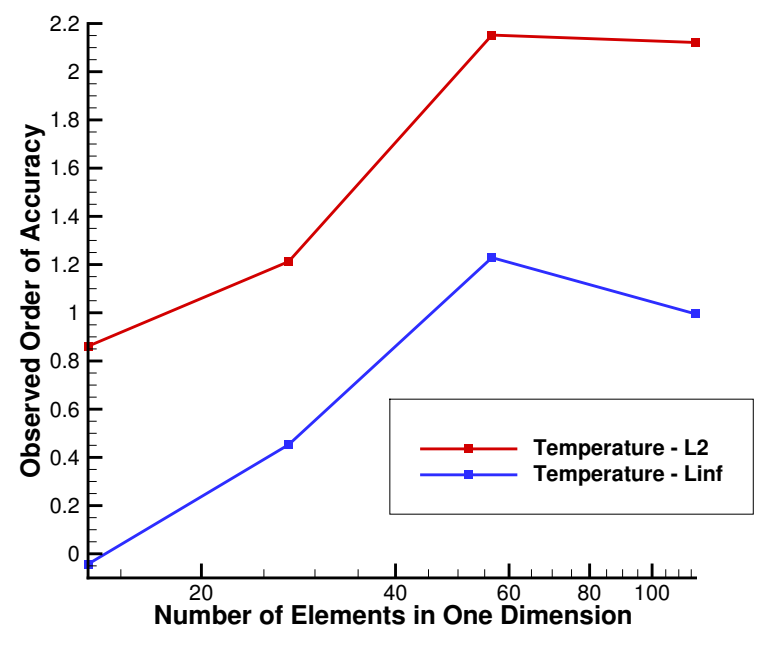

(b) Skewed domain

Figure 10: Observed order of accuracy of material response code at $t=1$ seconds with a specified flux boundary condition on one boundary. 
This again implies that errors which are less than second order are present at certain regions within the domain. Figure 11 supports this conclusion and shows that the errors are concentrated near the top corners of the domain where the specified flux and specified temperature boundary conditions meet. The reason for a concentration of errors at those points is still being investigated.

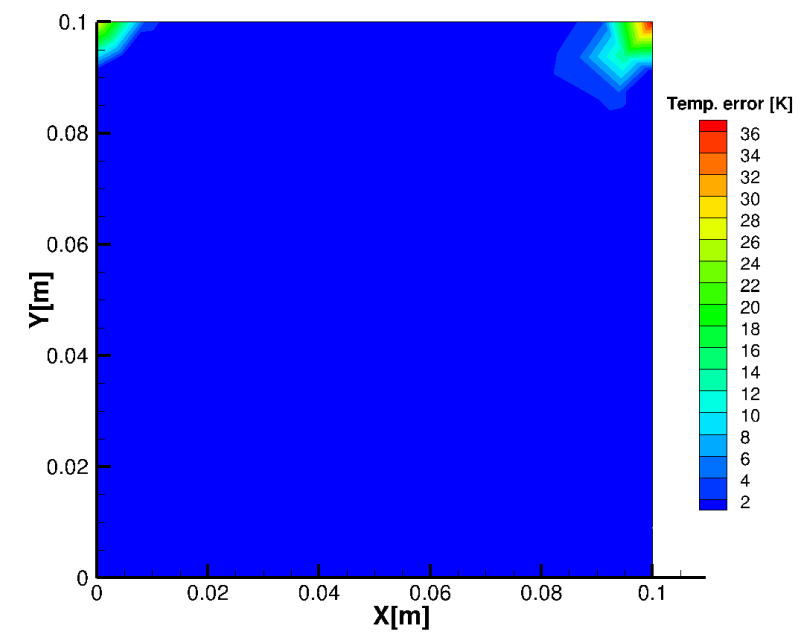

(a) Square domain

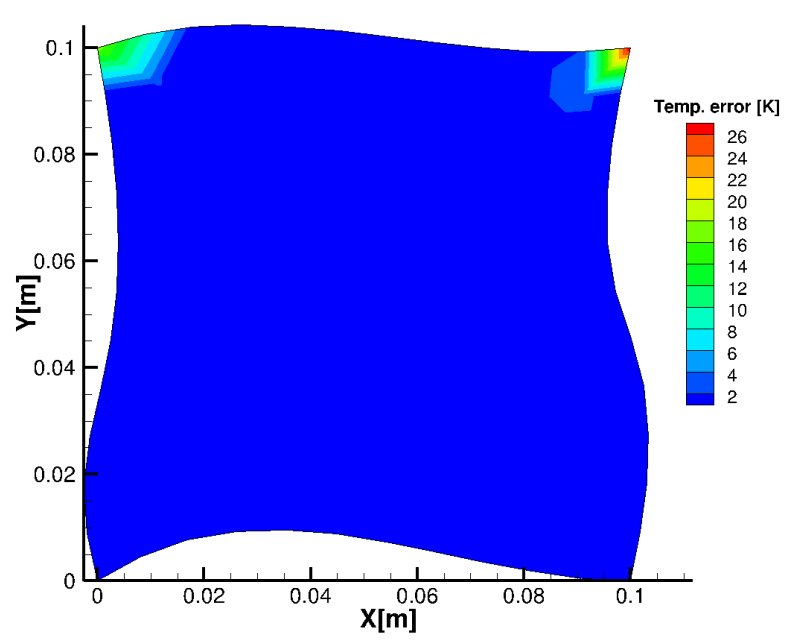

(b) Skewed domain

Figure 11: Error contours of temperature for the specified flux boundary condition case on two different domains.

\section{IV.C. Coupled Simulations}

Now that LeMANS and the material response code have been studied independently of one another, the effects on the order of accuracy of coupling them together are investigated. In this case, LeMANS is run with a manufactured solution and a wall boundary condition on the bottom boundary of the domain. The material response code is run without a manufactured solution, but using the heat flux values computed in LeMANS and fictitious material properties to compute an updated wall temperature. This wall temperature is then passed back to LeMANS and the $\epsilon$ terms in the manufactured solutions are updated to be consistent with the new temperature. In this case, $T_{t r}$ and $T_{v e}$ are set to be equal at the wall. An attempt is made to construct the manufactured solution and material properties in such a way that the computed wall temperature is constant along the boundary, but there are still slight variations in the wall temperature computed by the material response code. Therefore, the average wall temperature is the value actually passed back to LeMANS.

Figure 12 shows an example of the flow and solid domains that are used. To determine the order of accuracy, the flow field mesh is systematically refined, but the solid mesh remains constant. This decreases the number of variables that may impact the flow solution.

Figure 13 shows the results for the coupled simulations. While it is likely that one more mesh refinement is needed to reach the asymptotic regime, it can be seen that the order of accuracy results are quite similar to those shown in Figure 5 for the uncoupled wall boundary condition. However, the spread in order of accuracy for the different variables is much larger. It appears that all variables are going to converge to an order that is less than $2^{\text {nd }}$. Work is ongoing to pinpoint the cause. 


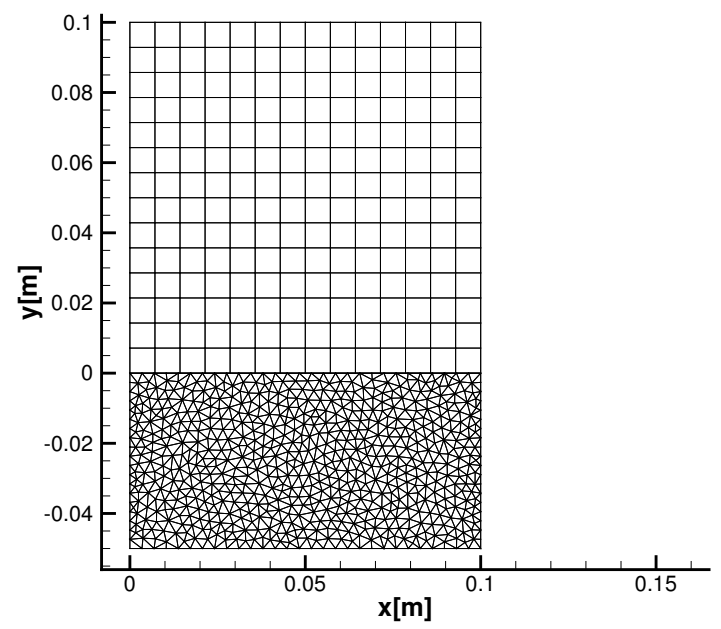

(a) Coarse flow mesh

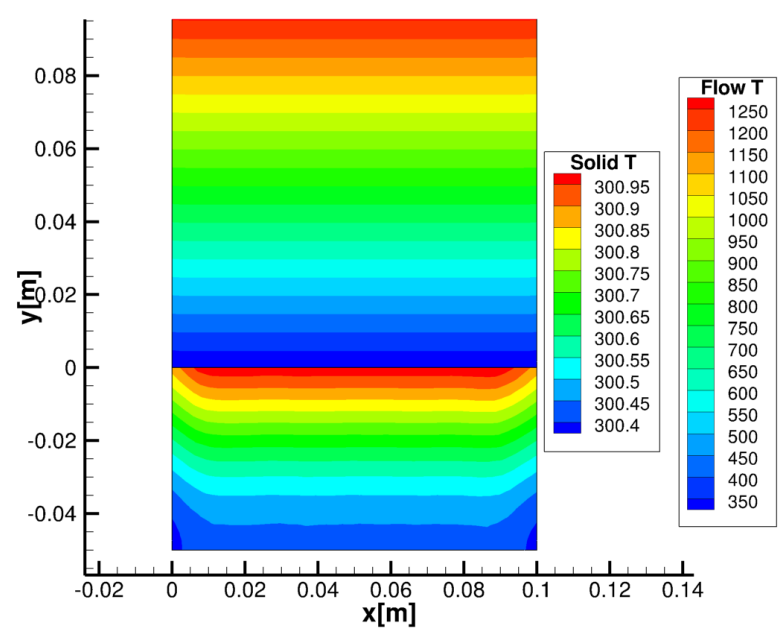

(b) Temperature contours on coarse flow mesh

Figure 12: Examples of coupled flow/solid domains.

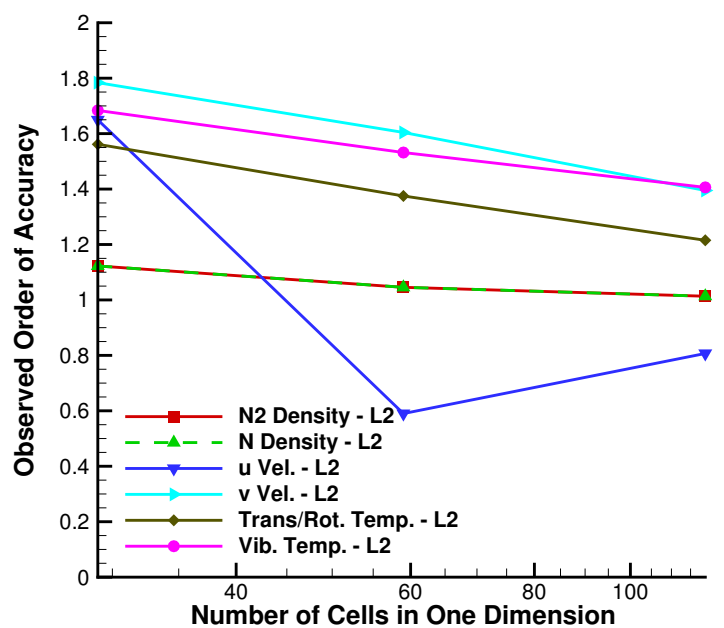

Figure 13: Observed order of accuracy based on the $L_{2}$ norm of the error for the coupled simulation. 


\section{Conclusions}

Using the Method of Manufactured Solutions, several aspects of two different codes were tested. The CFD code LeMANS was tested using both far field, supersonic outlet, and no-slip, isothermal boundary conditions. In all cases the observed order of accuracy was between $1^{\text {st }}$ and $2^{\text {nd }}$ order. The expected order of accuracy is $2^{\text {nd }}$; the lower observed order is still being investigated, but may be related to an implementation error with the MMS.

In addition to LeMANS, a material response code designed for the simulation of anisotropic materials was also verified using manufactured solutions. In this case, both specified temperature and specified heat flux boundary conditions were tested. In all tested cases, the expected $2^{\text {nd }}$ order spatial accuracy and $1^{\text {st }}$ order temporal accuracy were observed in the $L_{2}$ norm. In the specified flux cases, however, the $L_{\infty}$ norm showed a much lower order of accuracy, implying that lower order error is being generated in some portion of the domain. This is still being investigated. In addition, an error was identified in the computation of the boundary normal vectors that was leading to inaccurate results, underlining the usefulness of the Method of Manufactured Solutions as a verification technique.

Finally, LeMANS and the material response code were run in a coupled manner with the heat flux based on a manufactured solution computed in LeMANS used as an input to the material response code. An updated wall temperature was then computed and passed back to LeMANS, and the manufactured solution was updated to reflect the new wall temperature. This process was convergent and stable, but the observed order of accuracy in LeMANS was again between $1^{\text {st }}$ and $2^{\text {nd }}$.

This work indicates the necessity of code verification, and also the usefulness of the Method of Manufactured Solutions in the verification process. More work still needs to be done to fully verify all aspects of LeMANS and the material response code, but the work presented here represents an important first step towards that goal. It also increases confidence in the coupling methodology between LeMANS and the material response code.

\section{Acknowledgments}

Funding is provided by the Air Force Research Laboratory (AFRL) through the Collaborative Center in Aeronautical Sciences (CCAS).

\section{References}

${ }^{1}$ Knupp, P. and Salari, K., Verification of Computer Codes in Computational Science and Engineering, Chapman \& Hall/CRC, 2003.

${ }^{2}$ Roy, C., McWherter-Payne, M., and Oberkampf, W., "Verification and Validation for Laminar Hypersonic Flowfields, Part 1: Verification," AIAA Journal, Vol. 41, No. 10, 2003, pp. 1934-1943.

${ }^{3}$ Scalabrin, L. D., Numerical Simulation of Weakly Ionized Hypersonic Flow Over Reentry Capsules, Ph.D. thesis, University of Michigan, 2007.

${ }^{4}$ Martin, A., Scalabrin, L. C., and Boyd, I. D., "High Performance Modeling of Atmospheric Re-entry Vehicles," Journal of Physics: Conference Series, Vol. 341, 2012, Article 012002.

${ }^{5}$ Holman, T. D. and Boyd, I. D., "Effects of Continuum Breakdown on the Surface Properties of a Hypersonic Sphere," Journal of Thermophysics and Heat Transfer, Vol. 23, 2009, pp. 660-673.

${ }^{6}$ Sutton, K. and Gnoffo, P., "Multi-Component Diffusion with Application to Computational Aerothermodynamics," AIAA/ASME Joint Thermophysics and Heat Transfer Conference, Albuquerque, NM, June 15-18 1998, AIAA paper 19982575.

${ }^{7}$ Martin, A. and Boyd, I. D., "Strongly coupled computation of material response and nonequilibrium flow for hypersonic ablation," 41st AIAA Thermophysics Conference, San Antonio, Texas, June 22-25 2009, AIAA paper 2009-3597.

${ }^{8}$ MacCormack, R. W. and Candler, G. V., "The Solution of the Navier-Stokes Equations Using Gauss-Seidel Line Relaxation," Computers and Fluids, Vol. 17, No. 1, 1989, pp. 135-150.

${ }^{9}$ Jawahar, P. and Kamath, H., "A High-Resolution Procedure for Euler and Navier-Stokes Computations on Unstructured Grids," Journal of Computational Physics, Vol. 164, 2000, pp. 165-203.

${ }^{10}$ Martin, A. and Boyd, I. D., "Simulation of pyrolysis gas within a thermal protection system," 40th AIAA Thermophysics Conference, Seattle, Washington, June 23-26 2008, AIAA paper 2008-3805.

${ }^{11}$ Saad, Y. and Schultz, M. H., "GMRES: A Generalized Minimal Residual Algorithm for Solving Nonsymmetric Linear Systems," SIAM Journal on Scientific and Statistical Computing, Vol. 7, No. 3, July 1986, pp. 856-869.

${ }^{12}$ Amar, A. J., Modeling of One-Dimensional Ablation with Porous Flow Using Finite Control Volume Procedure, Master's thesis, North Carolina State University, 2006.

${ }^{13}$ Blackwell, B. and Hogan, R., "Numerical Solution of Axisymmetric Heat Conduction Problems Using Finite Control Volume Technique," Journal of Thermophysics and Heat Transfer, Vol. 7, No. 3, 1993, pp. 462-471. 
${ }^{14}$ Alkandry, H., Farbar, E. D., and Boyd, I. D., "Evaluation of Finite-Rate Surface Chemistry Models for Simulation of the Stardust Reentry Capsule," 43rd AIAA Thermophysics Conference, New Orleans, Louisiana, June 25-28 2012, AIAA paper 2012-2874.

${ }^{15}$ Hogan, R., Blackwell, B., and Cochran, R., "Application of Moving Grid Control Volume Finite Element Method to Ablation Problems," Journal of Thermophysics and Heat Transfer, Vol. 10, No. 2, April-June 1996, pp. 312-319.

${ }^{16}$ Wiebenga, J. E. and Boyd, I. D., "Computation of Multi-Dimensional Material Response Coupled to Hypersonic Flow," 43rd AIAA Thermophysics Conference, New Orleans, LA, June 25-28 2012, AIAA paper 2012-2873.

${ }^{17}$ Kuntz, D. W., Hassan, B., and Potter, D. L., "Predictions of Ablating Hypersonic Vehicles Using an Iterative Coupled Fluid/Thermal Approach," Journal of Thermophysics and Heat Transfer, Vol. 15, No. 2, 2001, pp. 129-139.

${ }^{18}$ Shepard, D., "A two-dimensional interpolation function for irregularly-spaced data," Proceedings of the 1968 23rd ACM national conference, ACM '68, ACM, New York, NY, USA, 1968, pp. 517-524.

${ }^{19}$ Franke, R., "Scattered Data Interpolation: Tests of Some Methods," Mathematics of Computation, Vol. 38, No. 157, January 1982.

${ }^{20}$ Amar, A., Calvert, N., and Kirk, B., "Development and Verification of the Charring Ablating Thermal Protection Implicit System Solver," 49th AIAA Aerospace Sciences Meeting including the New Horizons Forum and Aerospace Exposition, Orlando, Florida, January 4-7 2011, AIAA paper 2011-144.

${ }^{21}$ Veluri, S., Roy, C., Hebert, S., and Luke, E., "Verification of the Loci-CHEM CFD Code using the Method of Manufactured Solutions," 46th AIAA Aerospace Sciences Meeting and Exhibit, Reno, NV, January 7-10 2008, AIAA paper $2008-661$.

${ }^{22}$ Bond, R., Knupp, P., and Ober, C., "A Manufactured Solution for Verifying CFD Boundary Conditions," 34th AIAA Fluid Dynamics Conference and Exhibit, Portland, Oregon, June 28 - July 1 2004, AIAA paper 2004-2029.

${ }^{23}$ Bond, R., Knupp, P., and Ober, C., "A Manufactured Solution for Verifying CFD Boundary Conditions, Part II," 43rd AIAA Aerospace Sciences Meeting and Exhibit, Reno, NV, January 10-13 2005, AIAA paper 2005-88.

${ }^{24}$ Wolfram Research, I., Mathematica, Wolfram Research, Inc., version 8.0 ed., 2010. 